Document downloaded from:

http://hdl.handle.net/10251/51307

This paper must be cited as:

Lucas Alba, S. (2014). Using Representation Theorems for Proving Polynomials Nonnegative. En Artificial Intelligence and Symbolic Computation: 12th International Conference, AISC 2014, Seville, Spain, December 11-13, 2014. Proceedings. Springer Verlag (Germany). 21-33. doi:10.1007/978-3-319-13770-4_4.

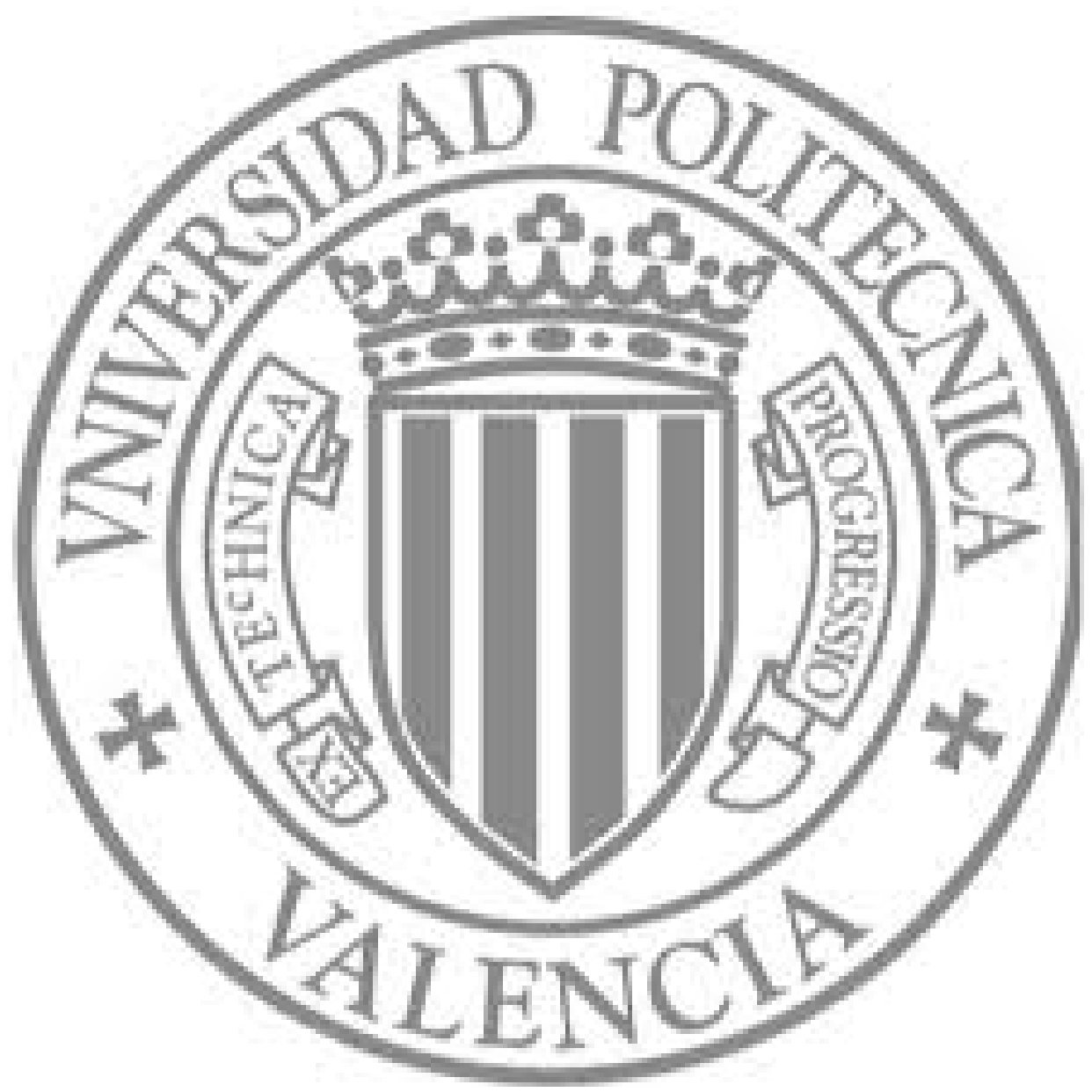

The final publication is available at

http://link.springer.com/chapter/10.1007/978-3-319-13770-4_4

Copyright Springer Verlag (Germany) 


\title{
Using Representation Theorems for Proving Polynomials Non-negative
}

\author{
Salvador Lucas ${ }^{1}$ \\ DSIC, Universidad Politécnica de Valencia \\ Camino de Vera s/n, 46022 Valencia, Spain
}

\begin{abstract}
Proving polynomials non-negative when variables range on a subset of numbers (e.g., $[0,+\infty))$ is often required in many applications (e.g., in the analysis of program termination). Several representations for univariate polynomials $P$ that are non-negative on $[0,+\infty)$ have been investigated. They can often be used to characterize the property, thus providing a method for checking it by trying a match of $P$ against the representation. We introduce a new characterization based on viewing polynomials $P$ as vectors, and find the appropriate polynomial basis $\mathcal{B}$ in which the non-negativeness of the coordinates $[P]_{\mathcal{B}}$ representing $P$ in $\mathcal{B}$ witnesses that $P$ is non-negative on $[0,+\infty)$. Matching a polynomial against a representation provides a way to transform universal sentences $\forall x \in[0,+\infty) P(x) \geq 0$ into a constraint solving problem which can be solved by using efficient methods. We consider different approaches to solve both kind of problems and provide a quantitative evaluation of performance that points to an early result by Pólya and Szegö's as an appropriate basis for implementations in most cases.
\end{abstract}

Keywords: Polynomial constraints, positive polynomials, representation theorems.

\section{Introduction}

Representations of univariate polynomials that are positive $(P d(I))$ or nonnegative $(P s d(I))$ on an interval $I$ of real numbers have been investigated (see [14] for a survey) and some of them are useful to check the property. In this paper we investigate this question: which technique is worth to be implemented for a practical use? Our specific motivation is the development of efficient and automatic tools for proving termination of programs, where polynomials play a prominent role (see $[8,12]$, for instance) and the focus is on $\operatorname{Psd}([0,+\infty)$ ).

We decompose the whole problem into two main steps: (1) the use of representation theorems to obtain a set of existential constraints whose satisfaction witnesses that $(\forall x \geq 0) P \geq 0$ holds and (2) the use of constraint solving techniques to obtain appropriate solutions. With regard to (1), several researchers (starting with Hilbert) addressed this problem and contributed in different ways (see Section 2). In this setting, the following test is often used in practice [10]: a polynomial $P$ is $\operatorname{Psd}\left([0,+\infty)^{n}\right)$ if all coefficients of the monomials in $P$ are nonnegative. This has obvious limitations. For instance, $Q(x)=x^{3}-4 x^{2}+6 x+1$ 
is $\operatorname{Psd}([0,+\infty))$, but contains negative coefficients. The following observation generalizes this approach (Section 3): $P \in \mathbb{R}[X]$ of degree $n$ can be represented as a vector $[P]_{\mathcal{B}}=\left(\alpha_{0}, \ldots, \alpha_{n}\right)^{T}$ of $n+1$ coordinates with respect to a $b a$ sis $\mathcal{B}=\left\{v_{0}, \ldots, v_{n}\right\} \subseteq \mathbb{R}[X]$, i.e., $P=\alpha_{0} v_{0}+\alpha_{1} v_{1}+\cdots+\alpha_{n} v_{n}$. Then, $P$ is $\operatorname{Psd}([0,+\infty))$ if (i) $[P]_{\mathcal{B}} \geq \mathbf{0}$ and $(i i) v_{0}, \ldots, v_{n}$ are $\operatorname{Psd}([0,+\infty))$. Requiring all coefficients in the representation $P=\sum_{i=0}^{n} p_{i} x^{i}$ to be non-negative corresponds to considering the standard basis $\mathcal{S}_{n}=\left\{1, x, \ldots, x^{n}\right\}$ for polynomials of degree $n$. In our running example, $[Q]_{\mathcal{S}_{3}}=(1,6,-4,1)^{T} \nsucceq \mathbf{0}$. We define a parametric polynomial basis $\mathcal{P}_{n}$ such that, for all $P \in \mathbb{R}[X]$ of degree $n$ which is $\operatorname{Psd}([0,+\infty))$, $[P]_{\mathcal{B}} \geq \mathbf{0}$ for some specific $\mathcal{B}$ which is obtained from $\mathcal{P}_{n}$ by giving appropriate values to the parameters. We also show how to give value to the parameters.

Example 1. The representation of $Q(x)=x^{3}-4 x^{2}+6 x+1$ with respect to $\mathcal{B}=\left\{1, x, x^{2}, x(x-2)^{2}\right\}$ is $[Q]_{\mathcal{B}}=(1,2,0,1)^{T} \geq \mathbf{0}$.

Regarding (2), in Section 4 we use a recent, efficient procedure to solve polynomial constraints over finite domains [5] as a reference to provide a quantitative analysis of the characterizations discussed in Sections 2 and 3 and provide an answer to our question. Section 5 discusses some related work and concludes.

\section{Representation of polynomials non-negative in $[0,+\infty)$}

We consider the following representations of $\operatorname{Psd}([0,+\infty))$ polynomials $P$ (see [14]): (1) Hilbert [9]; (2) Pólya and Szegö [13]; (3) Karlin and Studden [11]; and (4) Hilbert's approach using Gram matrices [7].

Remark 1. Our motivation for considering these particular methods is that, in automatic proofs of termination, polynomials $P$ whose non-negativity must be guaranteed are parametric, i.e., the coefficients are not numbers but rather variables whose value is generated by a constraint solving process. All previous methods fit the requirement of being amenable to this practical setting.

We briefly discuss how to use these four methods and also give some cost indicators: $V(n)$ is the number of parameters used to match $P$ (of degree $n$ ) against the representation, and $I(n)$ is the number of (in)equalities which are obtained. The following fact is used later.

Proposition 1. Let $P, Q \in \mathbb{R}\left[X_{1}, \ldots, X_{n}\right]$ be $P=\sum_{\alpha} a_{\alpha} X^{\alpha}$ and $Q=\sum_{\alpha} b_{\alpha} X^{\alpha}$. If $a_{\alpha} \geq b_{\alpha}$ for all $\alpha \in \mathbb{N}^{n}$ and $Q$ is Psd $\left([0,+\infty)^{n}\right)$, then $P$ is $\operatorname{Psd}\left([0,+\infty)^{n}\right)$.

In the following, $\div$ and $\%$ denote the integer division and remainder, respectively. We say that a polynomial $P$ is a sum of squares (or just sos, often denoted as $P \in \sum \mathbb{R}[\boldsymbol{X}]^{2}$ ) if can be written $P=\sum_{i} f_{i}^{2}$ for polynomials $f_{i}$.

\subsection{Hilbert}

Since $P \in \mathbb{R}\left[X_{1}, \ldots, X_{n}\right]$ is $\operatorname{Psd}\left([0,+\infty)^{n}\right)$ if and only if $H\left(X_{1}, \ldots, X_{n}\right)=$ $P\left(X_{1}^{2}, \ldots, X_{n}^{2}\right)$ is $\operatorname{Psd}\left(\mathbb{R}^{n}\right)$ (note that this transformation doubles the degree of $P$ ), we can use the following result. 
Proposition 2 (Hilbert). [9] If $P \in \mathbb{R}[X]$ is $P$ sd $(\mathbb{R})$, then $P$ is a sum of two squares of polynomials.

Example 2. Consider $H(x)=Q\left(x^{2}\right)=x^{6}-4 x^{4}+6 x^{2}+1=f_{1}(x)+f_{2}(x)$ where $f_{i}(x)=\left(a_{i} x^{3}+b_{i} x^{2}+c_{i} x+d_{i}\right)^{2}$ for $i=1,2$. Then, $H(x)$ should match

$\sum_{i=1}^{2} a_{i}^{2} x^{6}+2 a_{i} b_{i} x^{5}+\left(b_{i}^{2}+2 a_{i} c_{i}\right) x^{4}+2\left(b_{i} c_{i}+a_{i} d_{i}\right) x^{3}+\left(2 b_{i} d_{i}+c_{i}^{2}\right) x^{2}+2 c_{i} d_{i} x+d_{i}^{2}$

which amounts at solving the following equalities:

$$
\begin{aligned}
\sum_{i=1}^{2} a_{i}^{2} & =1 & \sum_{i=1}^{2} a_{i} b_{i} & =0 & \sum_{i=1}^{2} b_{i}^{2}+2 a_{i} c_{i} & =-4 \\
\sum_{i=1}^{2} b_{i} c_{i}+a_{i} d_{i} & =0 & \sum_{i=1}^{2} 2 b_{i} d_{i}+c_{i}^{2} & =6 & \sum_{i=1}^{2} c_{i} d_{i} & =0
\end{aligned}
$$

A solution (with irrational numbers) is obtained by using, e.g., Mathematica.

We have $V(n)=2 n+2$ and $I(n)=2 n+1$.

\subsection{Pólya and Szegö}

Proposition 3 (Pólya \& Szegö). [13] If $P$ is $P s d([0,+\infty))$, then there are sos polynomials $f, g$ such that $P(x)=f(x)+x g(x)$ and $\operatorname{deg}(f), \operatorname{deg}(x g) \leq \operatorname{deg}(P)$.

If $f, g \in \sum \mathbb{R}[X]^{2}$, then both $f$ and $x g$ are $P s d([0,+\infty))$. Thus, Pólya and Szegö's representation actually provides a characterization. We can use it, then, to prove that $P$ is $P s d([0,+\infty))$ iff $P$ matches the representation. Since every univariate sos polynomial $f$ can be written as a sum of two squares of polynomials, in Proposition 3 we assume $f=f_{1}^{2}+f_{2}^{2}$ and $g=g_{1}^{2}+g_{2}^{2}$, for polynomials $f_{i}$ and $g_{i}$, $i=1,2$. If $n=\operatorname{deg}(P)=1$, then, since $\operatorname{deg}(f), \operatorname{deg}(x g) \leq 1, f, g \in \sum \mathbb{R}[X]^{2}$ must be constant polynomials $f=f_{0}$ and $g=g_{0}$. If $n=2$, then, since $\operatorname{deg}(x g) \leq 2$, $g \in \sum \mathbb{R}[X]^{2}$ must be a constant. If $n>2$, then $\operatorname{deg}\left(f_{i}\right)=d_{1} \leq\left\lfloor\frac{n}{2}\right\rfloor$, and $\operatorname{deg}\left(g_{i}\right)=d_{2} \leq\left\lfloor\frac{n-1}{2}\right\rfloor$. Write $f_{i}=a_{i, d_{1}} x^{d_{1}}+\cdots+a_{i, 1} x+a_{i, 0}$ and $g_{i}=b_{i, d_{2}} x^{d_{2}}+$ $\cdots+b_{i, 1} x+b_{i, 0}$ for $i=1,2$. Try to match the coefficients of the target polynomial $P$ against this representation.

Example 3. For our running example $Q$, we have

$$
Q(x)=x^{3}-4 x^{2}+6 x+1=f_{1}(x)+f_{2}(x)+x\left(g_{1}(x)+g_{2}(x)\right)
$$

where $f_{i}(x)=\left(a_{i} x+b_{i}\right)^{2}$ and $g_{i}(x)=\left(c_{i} x+d_{i}\right)^{2}$ for $i=1,2$. Then,

$Q(x)=\left(c_{1}^{2}+c_{2}^{2}\right) x^{3}+\left(a_{1}^{2}+a_{2}^{2}+2 c_{1} d_{1}+2 c_{2} d_{2}\right) x^{2}+\left(2 a_{1} b_{1}+2 a_{2} b_{2}+d_{1}^{2}+d_{2}^{2}\right) x+b_{1}^{2}+b_{2}^{2}$

By Proposition 1, rather than equalities, we solve now the inequalities ${ }^{1}$ :

$1 \geq c_{1}^{2}+c_{2}^{2} ; \quad-4 \geq a_{1}^{2}+a_{2}^{2}+2 c_{1} d_{1}+2 c_{2} d_{2} ; 6 \geq 2 a_{1} b_{1}+2 a_{2} b_{2}+d_{1}^{2}+d_{2}^{2} ; 1 \geq b_{1}^{2}+b_{2}^{2}$.

with: $a_{1}=0, a_{2}=0, b_{1}=1, b_{2}=0, c_{1}=1, c_{2}=0, d_{1}=-2$, and $d_{2}=1$.

Each $f_{i}$ and $g_{i}$ contributes with $d_{1}+1$ and $d_{2}+1$ parametric coefficients, respectively, i.e., $V(n)=2\left(d_{1}+1+d_{2}+1\right)=2\left(2+d_{1}+d_{2}\right)=2(n+1)=2 n+2$. The number of inequalities to be solved is $I(n)=n+1$ (one per coefficient $p_{i}$ of $P$ ).

\footnotetext{
${ }^{1}$ Using inequalities makes the constraint solving process more flexible and often avoids
} the use of irrational numbers, often out of the scope for most constraint solving tools. 


\subsection{Karlin and Studden}

Theorem 1 (Karlin and Studden). [11, Corollary V.8.1] Let $P_{2 m}$ be a polynomial of degree $2 m$ for some $m \geq 0$ with leading coefficient $a_{2 m}>0$. If $P_{2 m}$ is $P d([0,+\infty))$, then there exists a unique representation

$$
P_{2 m}(X)=a_{2 m} \prod_{j=1}^{m}\left(X-\alpha_{j}\right)^{2}+\beta X \prod_{j=2}^{m}\left(X-\gamma_{j}\right)^{2}
$$

where $\beta>0$ and $0=\gamma_{1}<\alpha_{1}<\gamma_{2}<\cdots<\gamma_{m}<\alpha_{m}<\infty$. Similarly, if $P_{2 m+1}$ is a polynomial of degree $2 m+1$ for some $m \geq 0$, with leading coefficient $a_{2 m+1}>0$ and $P_{2 m+1}$ is $\operatorname{Pd}([0,+\infty))$, then there exists a unique representation

$$
P_{2 m+1}(X)=a_{2 m+1} X \prod_{j=2}^{m+1}\left(X-\alpha_{j}\right)^{2}+\beta \prod_{j=1}^{m}\left(X-\gamma_{j}\right)^{2}
$$

where $\beta>0$ and $0=\alpha_{1}<\gamma_{1}<\alpha_{2}<\gamma_{2}<\cdots<\gamma_{m}<\alpha_{m+1}<\infty$.

Unfortunately, this representation cannot be used to prove that $P$ is $P d([0,+\infty))$ by matching. For instance, $P=(x-1)^{2}$ matches it, but it is not $P d([0,+\infty))$. However, Karlin and Studden's representation can be used to prove $P$ to be $\operatorname{Psd}([0,+\infty))$ by matching if we just require $\alpha_{j}, \beta, \gamma_{j} \geq 0$.

Example 4. Since the degree of $Q$ is odd, we let

$$
K_{Q}(x)=x\left(x-\alpha_{2}\right)^{2}+\beta\left(x-\gamma_{1}\right)^{2}=x^{3}+\left(\beta-2 \alpha_{2}\right) x^{2}+\left(\alpha_{2}^{2}-2 \beta \gamma_{1}\right) x+\beta \gamma_{1}^{2}
$$

Thus, we have the following constraints (using Proposition 1):

$$
-4 \geq \beta-2 \alpha_{2} \quad 1 \geq 0 \quad 6 \geq \alpha_{2}^{2}-2 \beta \gamma_{1} \quad 1 \geq \beta \gamma_{1}^{2} \quad \beta \geq 0 \quad \gamma_{1} \geq 0 \quad \alpha_{2} \geq 0
$$

The assignment $\alpha_{2}=\frac{9}{4}, \beta=\frac{1}{4}$, and $\gamma_{1}=\frac{1}{2}$ solves the system.

We have $V(n)=n$ and $I(n)=n+1+V(n)=2 n+1$.

\subsection{Hilbert with Gram matrices}

An alternative way to use Hilbert's representation is the following.

Theorem 2. [7] Let $P$ be a polynomial of degree $2 m$ and $z(X)$ be the vector of all monomials $X^{\alpha}$ such that $|\alpha| \leq m$. Then, $P$ is a sum of squares in $\mathbb{R}[X]$ if and only if there exists a real, symmetric, psd matrix $B$ such that $P=z(X)^{T} B z(X)$.

Proving $H(x)=P\left(x^{2}\right)$ of degree $2 n$ to be sos amounts at (1) matching $H$ against $z(X)^{T} B z(X)$ (where $z(X)=\left(1, X, \ldots, X^{n}\right)^{T}$ ) and (2) proving $B \in \mathbb{R}^{n+1 \times n+1}$ positive semidefinite. Since $B$ is symmetric, we need $\frac{(n+1)(n+2)}{2}$ parameters $b_{i j}$ to represent $B$. Then, we need to solve $2 n+1$ equations in $\frac{(n+1)(n+2)}{2}$ variables (the parameters $b_{i j}$ ) corresponding to the monomials in $H$. According to [15], this can be done by taking $\frac{(n+1)(n+2)}{2}-(2 n+1)=\frac{n^{2}-n}{2}$ of the $b_{i j}$ as unknowns which can be given appropriate values that are obtained using (2), i.e., $B$ must be positive semidefinite. This can be done by computing the characteristic polynomial $\operatorname{det}\left(z I_{n+1}-B\right)=\sum_{i=0}^{n} c_{i} z^{i}$ of $B$ and requiring its roots to be non-negative [15]. They show that this can be achieved by imposing $(-1)^{i+n+1} c_{i} \geq 0$ for all $0 \leq i \leq n$. Thus, $V(n)=\frac{(n+1)(n+2)}{2}$. and $I(n)=(2 n+1)+(n+1)=3 n+2$. 


\section{Checking positiveness of polynomials as vectors}

Let $\mathrm{V}$ be an $n$-dimensional vector space over the reals and $\mathcal{B}=\left\{v_{1}, \ldots, v_{n}\right\}$ be an ordered basis for $\mathrm{V}$. For all $n$-tuples $\boldsymbol{\alpha}=\left(\alpha_{1}, \ldots, \alpha_{n}\right) \in \mathbb{R}^{n}$ we write $\boldsymbol{\alpha} \geq 0$ if $\alpha_{i} \geq 0$ and $\boldsymbol{\alpha}>0$ if $\alpha_{1}>0$ and $\alpha_{2}, \ldots, \alpha_{n} \geq 0$. Every $\boldsymbol{v} \in \mathrm{V}$ can be represented as a coordinate vector $[\boldsymbol{v}]_{\mathcal{B}}=\left(\alpha_{1}, \ldots, \alpha_{n}\right)^{T} \in \mathbb{R}^{n}$ such that $\boldsymbol{v}=\alpha_{1} v_{1}+\cdots+\alpha_{n} v_{n}$. Given bases $\mathcal{B}$ and $\mathcal{B}^{\prime}$ for $\mathrm{V}$, there is a change of base matrix (cb-matrix) $M_{\mathcal{B}^{\prime} \mapsto \mathcal{B}}$ (or just $M$ ) which can be used to obtain the coordinate representation $[\boldsymbol{v}]_{\mathcal{B}}$ of $\boldsymbol{v}$ in $\mathcal{B}$ from the representation $[\boldsymbol{v}]_{\mathcal{B}^{\prime}}$ of $\boldsymbol{v}$ in $\mathcal{B}^{\prime}:[\boldsymbol{v}]_{\mathcal{B}}=$ $M[\boldsymbol{v}]_{\mathcal{B}^{\prime}}$. The set $\mathbb{P}_{n}$ of univariate polynomials of degree at most $n$ is a vectorial space of dimension $n+1$ and has a standard basis $\mathcal{S}_{n}=\left\{1, x, \ldots, x^{n}\right\}$. If $\mathcal{B}=$ $\left\{v_{0}, \ldots, v_{n}\right\}$ is a basis for $\mathbb{P}_{n}$ and every $v \in \mathcal{B}$ is $\operatorname{Psd}([0,+\infty))$, then given $P \in \mathbb{P}_{n}$, if $[P]_{\mathcal{B}}=\left(\alpha_{0}, \ldots, \alpha_{n}\right)^{T} \geq \mathbf{0}$, then $P$ is $\operatorname{Psd}([0,+\infty))$. If $P=\sum_{i=0}^{n} p_{i} x^{i}$, this is translated into the search of a basis $\mathcal{B}$ satisfying the conditions above and a $c b$-matrix $M=M_{\mathcal{S}_{n} \mapsto \mathcal{B}}$ such that $M[P]_{\mathcal{S}_{n}} \geq \mathbf{0}$. We consider parametric bases $\mathcal{B}$ consisting of polynomials with parametric coefficients which can be given appropriate values as to fit the requirements above. By a parametric polynomial we mean a polynomial $P \in \mathbb{R}\left[\gamma_{1}, \ldots, \gamma_{k}\right][X]$ over $X$ whose monomials have coefficients in $\mathbb{R}\left[\gamma_{1}, \ldots, \gamma_{k}\right]$; variables $\gamma_{1}, \ldots, \gamma_{k}$ are called parameters. For all $i \in \mathbb{N}$, consider the parametric univariate polynomials, :

$$
\mathrm{P}_{i}(x)=\prod_{j=1}^{\frac{i}{2}}\left(x-\gamma_{i j}\right)^{2} \text { if } i \text { is even } \quad \mathrm{P}_{i}(x)=x \prod_{j=1}^{\frac{i-1}{2}}\left(x-\gamma_{i j}\right)^{2} \text { if } i \text { is odd }
$$

where the empty product is 1 , and $\gamma_{i j}$ are parameters satisfying $\gamma_{i j} \geq 0$. For instance, $\mathrm{P}_{0}(x)=1, \mathrm{P}_{1}(x)=x, \mathrm{P}_{2}(x)=\left(x-\gamma_{21}\right)^{2}=\gamma_{21}^{2}-2 \gamma_{21} x+x^{2}$, and $\mathrm{P}_{3}(x)=x\left(x-\gamma_{31}\right)^{2}=\gamma_{31}^{2} x-2 \gamma_{31} x^{2}+x^{3}$. Note that for all $i \geq 0$ and $x \geq 0$, $\mathrm{P}_{i}(x) \geq 0$ and $\mathrm{P}_{0}(x)>0$. Given $n \in \mathbb{N}$, let $\mathcal{P}_{n}=\left\{\mathrm{P}_{0}(x), \ldots, \mathrm{P}_{n}(x)\right\}$ ordered by the sequence $0,1, \ldots, n$. $\mathcal{P}_{n}$ is a basis of $\mathbb{P}_{n}$; this is a consequence of the following.

Theorem 3. Let $\mathcal{P}=\left\{P_{0}, \ldots, P_{n}\right\}$ be a set of $n+1$ polynomials such that $P_{0} \in \mathbb{R}-\{0\}$ and $\operatorname{deg}\left(P_{i}\right)=i$ for all $1 \leq i \leq n$. Then, $\mathcal{P}$ is a basis of $\mathbb{P}_{n}(x)$.

Note that $\mathcal{P}_{n+1}=\mathcal{P}_{n} \cup \mathrm{P}_{n+1}(x)$.

Proposition 4 (Number of parameters in the basis). Given $n \in \mathbb{N}$, the number $N(n)$ of parameters in $\mathcal{P}_{n}$ is given by $N(0)=0$ and $N(n)=N(n-1)+$ $\left\lfloor\frac{n}{2}\right\rfloor$ for $n>0$. Furthermore, $N(n)=\frac{n^{2}}{4}$ if $n$ is even and $\frac{n^{2}-1}{4}$ otherwise.

We prove that $\mathcal{P}_{n}$ characterizes $\operatorname{Psd}([0,+\infty))$ and $\operatorname{Pd}([0,+\infty))$.

Theorem 4. A polynomial $P \in \mathbb{R}[X]$ of degree $n$ is $\operatorname{Psd}([0,+\infty))(\operatorname{Pd}([0,+\infty)))$ if and only if $[P]_{\mathcal{P}_{n}} \geq \mathbf{0}$ (resp. $[P]_{\mathcal{P}_{n}}>\mathbf{0}$ ) for some assignment of values $\gamma_{i j} \geq 0$ to the parameters in $\mathcal{P}_{n}$.

We show how to compute the cb-matrix $M_{n}=M_{\mathcal{S}_{n} \mapsto \mathcal{P}_{n}}$ for obtaining the representation $[P]_{\mathcal{P}_{n}}=M_{n}[P]_{\mathcal{S}_{n}}$ of $P \in \mathbb{P}_{n}$ which is required in Theorem 4 . In the following, $\left[\mathrm{P}_{n}(x)\right]_{\mathcal{S}_{n}}^{1, \cdots, n}$ is the $n$-dimensional vector containing the first $n$ (parametric) coordinates of $\left[\mathrm{P}_{n}(x)\right]_{\mathcal{S}_{n}}$ (the last one is 1 , corresponding to $x^{n}$ ). 
Theorem 5 (Incremental cb-matrix). We have $M_{0}=I_{1}$ and for all $n>0$,

$$
M_{n}=\left(\begin{array}{cc}
M_{n-1} & -M_{n-1}\left[\mathrm{P}_{n}(x)\right]_{\mathcal{S}_{n}}^{1, \ldots, n} \\
\mathbf{0}_{1 \times n} & 1
\end{array}\right)
$$

Example 5. Since $M_{1}=I_{2}$, according to Theorem 5, we have:

$$
\begin{gathered}
M_{2}=\left(\begin{array}{cc}
M_{1} & -M_{1}\left(\begin{array}{c}
\gamma_{21}^{2} \\
-2 \gamma_{21}
\end{array}\right) \\
\mathbf{0}_{1 \times 2} & 1
\end{array}\right)=\left(\begin{array}{ccc}
1 & 0 & -\gamma_{21}^{2} \\
0 & 1 & 2 \gamma_{21} \\
0 & 0 & 1
\end{array}\right) \text { and } \\
M_{3}=\left(\begin{array}{cc}
0 \\
M_{2} & -M_{2}\left(\begin{array}{c}
0 \\
\gamma_{31}^{2} \\
-2 \gamma_{31}
\end{array}\right) \\
1 & \mathbf{0}_{1 \times 3}
\end{array}\right)=\left(\begin{array}{cccc}
1 & 0 & -\gamma_{21}^{2} & -2 \gamma_{21}^{2} \gamma_{31} \\
0 & 1 & 2 \gamma_{21} & 4 \gamma_{21} \gamma_{31}-\gamma_{31}^{2} \\
0 & 0 & 1 & 2 \gamma_{31} \\
0 & 0 & 0 & 1
\end{array}\right)
\end{gathered}
$$

For our running example $[Q]_{\mathcal{S}_{3}}=(1,6,-4,1)^{T}$, we impose $[Q]_{\mathcal{P}_{3}}=M_{3}[Q]_{\mathcal{S}_{3}}>0$ :

$$
\left(\begin{array}{cccc}
1 & 0 & -\gamma_{21}^{2} & -2 \gamma_{21}^{2} \gamma_{31} \\
0 & 1 & 2 \gamma_{21} & 4 \gamma_{21} \gamma_{31}-\gamma_{31}^{2} \\
0 & 0 & 1 & 2 \gamma_{31} \\
0 & 0 & 0 & 1
\end{array}\right)\left(\begin{array}{c}
1 \\
6 \\
-4 \\
1
\end{array}\right)=\left(\begin{array}{c}
1+4 \gamma_{21}^{2}-2 \gamma_{21}^{2} \gamma_{31} \\
6-8 \gamma_{21}+4 \gamma_{21} \gamma_{31}-\gamma_{31}^{2} \\
-4+2 \gamma_{31} \\
1
\end{array}\right)>\left(\begin{array}{l}
0 \\
0 \\
0 \\
0
\end{array}\right)
$$

The corresponding existential constraint:

$\gamma_{21}, \gamma_{31} \geq 0,1+4 \gamma_{21}^{2}-2 \gamma_{21}^{2} \gamma_{31}>0 \wedge 6-8 \gamma_{21}+4 \gamma_{21} \gamma_{31}-\gamma_{31}^{2} \geq 0 \wedge 2 \gamma_{31}-4 \geq 0 \wedge 1>0$ is satisfied if $\gamma_{21}=0$ and $\gamma_{31}=2$, witnessing $Q$ as $p d([0,+\infty))$ through the coordinate representation $[Q]_{\mathcal{P}_{3}}=(1,2,0,1)^{T}$ when $\mathcal{P}_{3}=\left\{1, x, x^{2}, x(x-2)^{2}\right\}$.

Note that $V(n)=N(n)=\frac{n^{2}-n \% 2}{4}$ and $I(n)=n+1+V(n)=n+1+\frac{n^{2}-n \% 2}{4}$.

Remark 2. If $P$ is a parametric polynomial of degree $n$, then $[P]_{\mathcal{S}_{n}}$ is an $n+1$ tuple of parameters which are treated by the constraint solving system which obtains the parameters of the basis $\mathcal{P}_{n}$ in the same way (see Remark 1).

\section{Quantitative analysis}

In constraint solving, the number of variables occurring in the whole set of constraints usually dominates the temporal cost to reach a solution. In our setting, assuming $P$ of degree $n$, for each representation method $V(n)$ and $I(n)$ (see Section 2) are as follows:

\begin{tabular}{|c|c|c|c|c|c|}
\hline Method: & Hilbert & P\&S & K\&S & Gram & Vector \\
\hline$V(n):$ & $2 n+2$ & $2 n+2$ & $n+1$ & $\frac{(n+1)(n+2)}{2}$ & $\frac{n^{2}-n \% 2}{4}$ \\
$I(n):$ & $2 n+1$ & $n+1$ & $2 n+1$ & $3 \mathrm{n}+2$ & $n+1+\frac{n^{2}-n \% 2}{4}$ \\
\hline
\end{tabular}

This table suggests the following conclusion: for proving Psd $([0,+\infty))$, Karlin $\&$ Studden is the best choice. However, this does not pay attention to the subsequent constraint solving process that we need to use in any implementation. In [5] an 
efficient procedure to solve polynomial constraints $C$ (e.g., $P \geq 0$, where $P$ is written as a sum of monomials with the corresponding coefficients) is given. The procedure transforms a polynomial constraint into a formula of the linear arithmetic and then fast, highly efficient Satisfiability Modulo Theories (SMT) techniques are used to find a solution. In linear arithmetic (logic) only constants $c$ or additions of linear expressions $c \cdot v$ are allowed, the atoms consist of expressions $\ell \bowtie \ell^{\prime}$ where $\ell, \ell^{\prime}$ are constants or linear expressions and $\bowtie \in\{=,>, \geq\}$, and the formulas are combinations of atoms using $\rightarrow$ (implication) and $\wedge$ (conjunction). An initial preprocessing $L 0$ transforms $P \bowtie 0$ into $\ell_{P} \bowtie 0$, where $\ell_{P}$ is obtained from $P$ by replacing the nonlinear monomials $M$ by new variables $x_{M}$; then new atoms $x_{M}=M$ are added and they are subsequently transformed after further linearization using the following rules, where $D$ is a finite domain of numbers ${ }^{2}$ :

Definition 1. Let $C$ be a pure non-linear constraint and $D$ be a finite set. The transformation rules are the following (where $v$ is a variable):

$$
\begin{aligned}
& \text { L1: } C \wedge x=v^{p} \Longrightarrow C \wedge \bigwedge_{a \in D}\left(v=a \rightarrow x=a^{p}\right) \text {, if } p>1 \\
& \text { L2: } C \wedge x=v^{p} \cdot w \Longrightarrow C \wedge \bigwedge_{a \in D}\left(v=a \rightarrow x=a^{p} \cdot w\right) \\
& \text { L3: } C \wedge x=v^{p} \cdot M \Longrightarrow C \wedge \bigwedge_{a \in D}\left(v=a \rightarrow x=a^{p} \cdot x_{M}\right) \wedge x_{M}=M \\
& \quad \text { if } M \text { is not linear and } v \text { does not occur in } M
\end{aligned}
$$

For $x=M_{0}$ where $M_{0}$ is a monomial with $m$ different variables, if $M_{0}$ consists of at most two variables, one of them of degree 1 , then $L 1$ or $L 2$ apply; no new variables are introduced and the equality is transformed into $|D|$ new linear formulas. If $M_{0}=v^{p} M$ contains $m$ variables and $M$ is not linear, then only $L 3$ applies, and then introduces a new variable $x_{M}$ together with $|D|$ new linear formulas and a new equality $x_{M}=M$ where $M$ has $m-1$ variables.

Example 6. For instance, for $1 \geq c_{1}^{2}+c_{2}^{2}$ in Example 3,

$$
\begin{aligned}
& 1 \geq c_{1}^{2}+c_{2}^{2} \leadsto L 01 \geq x_{c_{1}^{2}}+x_{c_{2}^{2}} \wedge x_{c_{1}^{2}}=c_{1}^{2} \wedge x_{c_{2}^{2}}=c_{2}^{2} \\
& \quad \sim L 11 \geq x_{c_{1}^{2}}+x_{c_{2}^{2}} \wedge \bigwedge_{d \in D} c_{1}=d \rightarrow x_{c_{1}^{2}}=d^{2} \wedge \bigwedge_{d \in D} c_{2}=d \rightarrow x_{c_{2}^{2}}=d^{2}
\end{aligned}
$$

we obtain $1+2|D|$ linear formulas and 2 new variables are required.

In the following, $V_{L}(n)$ is the number of new variables introduced by $L 0$. And if $P$ is the targeted polynomial, $p_{i}$ for $0 \leq i \leq n$ is the coefficient of $x^{i}$ in $P$.

Hilbert. If $f=\sum_{j=0}^{d} f_{j} x^{j}$ is a parametric polynomial of degree $d>0$, then the coefficient $c_{i}$ of $x^{i}$ in $f^{2}$ is obtained from the products $f_{r} f_{s}$ such that $r+s=i$. Here, $f_{s} f_{r}$ does not count as a new combination because $f_{r} f_{s}+f_{s} f_{r}=2 f_{r} f_{s}$. If $i \leq d$ we have different contributing combinations from $(0, i)$ to $(i \div 2, i-i \div 2)$, i.e., $1+i \div 2$ combinations. If $i>d$, then we have different contributing combinations starting from $(d-i, d)$, i.e., $1+(2 d-i) \div 2=1+d-i \div 2-i \% 2$ combinations. Overall, if $\mu_{d}\left(c_{i}\right)=1+i \div 2$, if $i \leq d$, and $\mu_{d}\left(c_{i}\right)=1+d-i \div 2-i \% 2$, if $i>d$, then $c_{i}$ consists of a sum of $\mu_{d}\left(c_{i}\right)$ monomials $f_{r} f_{s}$ all of them of degree 2 .

\footnotetext{
${ }^{2}$ Simplified definition which only uses a single domain of values for all variables.
} 
When matching $P(x)=\sum_{i=0}^{n} p_{i} x_{i}^{i}$ against Hilbert's representation, each $p_{i}, 0 \leq i \leq n$ is matched by a sum $c_{2 i}$ of $2 \mu_{n}\left(c_{2 i}\right)$ expressions of degree 2 (in the parameters). However, for all $0 \leq i<n$, there are additional equations $c_{2 i+1}=0$ which are due to the duplication of the degree of $P$ before the matching. Therefore, there are $2 n+1$ equations gathering

$$
\left.\sum_{i=0}^{n} 2 \mu_{n}\left(c_{2 i}\right)+\sum_{i=0}^{n-1} 2 \mu_{n}\left(c_{2 i+1}\right)=2\left(\sum_{i=0}^{n} \mu_{n}\left(c_{2 i}\right)+\sum_{i=0}^{n-1} \mu_{n}\left(c_{2 i+1}\right)\right)\right)
$$

quadratic terms all together, i.e., $\left.V_{L}(n)=2\left(\sum_{i=0}^{n} \mu_{n}\left(c_{2 i}\right)+\sum_{i=0}^{n-1} \mu_{n}\left(c_{2 i+1}\right)\right)\right)$.

Polya \& Szegö. When matching $P=\sum_{i=0}^{n} p_{i} x_{i}^{i}$ against Polya and Szegö's representation in Section 2.2, if $n=1$, then $p_{0}$ and $p_{1}$ are matched to squared constants $f_{0}^{2}$ and $g_{0}^{2}$, respectively. If $n=2$, then $p_{1}$ is matched to a sum of two monomials of degree 2 each; finally, if $n \geq 3$, then $p_{0}$ and $p_{n}$ are each of them matched to a sum of 2 squares, and each $p_{i}, 0<i<n$ is matched to a sum of $2 \mu_{n \div 2}\left(c_{i}\right)+2 \mu_{(n-1) \div 2}\left(c_{i-1}\right)$ expressions which are parametric coefficients: the coefficients of monomials of degree $i$ from $f_{1}^{2}$ and $f_{2}^{2}$, and the coefficients of monomials of degree $i-1$ from $g_{1}^{2}$ and $g_{2}^{2}$. All these parametric coefficients have degree 2. We have two equations with two terms and $n-1$ equations gathering

$$
\begin{gathered}
\sum_{i=1}^{n-1} 2 \mu_{n \div 2}\left(c_{i}\right)+2 \mu_{(n-1) \div 2}\left(c_{i-1}\right)=2\left(\sum_{i=1}^{n-1} \mu_{n \div 2}\left(c_{i}\right)+\sum_{i=1}^{n-1} \mu_{(n-1) \div 2}\left(c_{i-1}\right)\right) \\
=2\left(1+\mu_{n \div 2}\left(c_{n-1}\right)+\sum_{i=1}^{n-2} \mu_{n \div 2}\left(c_{i}\right)+\mu_{(n-1) \div 2}\left(c_{i}\right)\right)
\end{gathered}
$$

terms. Terms $M$ of degree 2 require a new variable $x_{M}$ in the initial step $L 0$. Overall, $V_{L}(1)=2, V_{L}(2)=3 \cdot 2=6$ and, for $n \geq 3$ :

$$
V_{L}(n)=6+2\left(\mu_{n \div 2}\left(c_{n-1}\right)+\sum_{i=1}^{n-2} \mu_{n \div 2}\left(c_{i}\right)+\mu_{(n-1) \div 2}\left(c_{i}\right)\right)
$$

Karlin \& Studden. If $\alpha \in\{0, \ldots, n\}^{m}$, we let $|\alpha|=\sum_{i=1}^{m} \alpha_{i}$. Note that $\left(\prod_{i=1}^{m}\left(x-a_{i}\right)\right)^{n}=\sum_{i=0}^{m n}(-1)^{i}\left(\sum_{\alpha \in\{0, \ldots, n\}^{m},|\alpha|=m n-i} \boldsymbol{a}^{\alpha}\right) x^{i}$. If $n=1$, there are $\left(\begin{array}{c}m \\ m-i\end{array}\right)=\left(\begin{array}{c}m \\ i\end{array}\right)$ parametric monomials $\boldsymbol{a}^{\alpha}$ (all of them of degree $m-i$ with respect to parameters $a_{i}$ ) accompanying $x^{i}$. If $n=2$, we can obtain the number of monomials accompanying $x^{i}$ as follows. There are $\left(\begin{array}{c}m \\ p\end{array}\right)$ monomials $\boldsymbol{a}^{\alpha}$ with $\alpha \in\{0,1\}^{m}$ and $|\alpha|=m-p$. Here, $0 \leq p \leq m$. These monomials can contribute to a monomial of degree $2 m-i$ for $x^{i}$. However, note that only those monomials satisfying $m-p \leq 2 m-i$ (i.e., $p \geq i-m$ ) will be useful; otherwise, the monomials $\boldsymbol{a}^{\alpha}$ exceed the required degree $2 m-i$ for $x^{i}$. If we replace $2 m-i-(m-p)=$ $m-i+p$ occurrences of 1 by 2 in $\alpha$ to yield $\alpha^{\prime}$ (with $m-p-(m-i+p)=i-2 p$ occurrences of 1 only), then, $\left|\alpha^{\prime}\right|=2(m-i+p)+i-2 p=2 m-i$ as desired. We can do that in $\left(\begin{array}{c}m-p \\ m-i+p\end{array}\right)$ different ways. However, this process makes sense only 
if $\alpha$ has enough occurrences of 1 , i.e., if $2(m-p) \geq 2 m-i$ (equivalently, $2 p \leq i$, i.e., $p \leq i \div 2$ ) so that the replacement of occurrences of 1 by 2 in $\alpha$ actually leads to the appropriate $\alpha^{\prime}$. Overall, $x^{i}$ comes with a parametric coefficient of

$$
\operatorname{mon}(m, i)=\sum_{p=\max (0, i-m)}^{i \div 2}\left(\begin{array}{c}
m \\
p
\end{array}\right)\left(\begin{array}{c}
m-p \\
m-i+p
\end{array}\right)
$$

monomials of degree $2 m-i$ (in the parameters $a_{i}$ ).

When matching a polynomial $P$ of degree $2 m$ against Karlin \& Studden representation, we get $2 m+1$ constraints $C_{i} \leq p_{i}, 0 \leq i \leq 2 m$, where $C_{i}$ consists of mon $(m, i)$ monomials of degree $2 m-i$ (coming from the first term of $P_{2 m}(X)$ in Theorem 1$)$ and $\operatorname{mon}(m-1, i-1)$ monomials of degree $2 m-i$ (due to the product with $\beta$ and $X)$ coming from the second term of $P_{2 m}(X)$. Therefore, $C_{i}$ consists of nonlinear monomials if $2 m-i>1$ (i.e., $i<2 m-1$ ). Overall, we have $\sum_{i=0}^{2 m-2}(\operatorname{mon}(m, i)+\operatorname{mon}(m-1, i-1))$ nonlinear monomials. Similarly, $P$ of degree $2 m+1$ yields $2 m+2$ constraints $C_{i}=p_{i}, 0 \leq i \leq 2 m+1$, where $C_{i}$ consists of $\operatorname{mon}(m, i-1)$ monomials of degree $2 m-i+1$ (coming from the first term of $P_{2 m}(X)$ above) and $\operatorname{mon}(m, i)$ monomials of degree $2 m-i+1$ (due to the product with $\beta$ ) coming from the second term of $P_{2 m}(X)$. Therefore, $C_{i}$ consists of nonlinear monomials if $2 m-i+1>1$ (i.e., $i<2 m$ ). Overall, $\sum_{i=0}^{2 m-1}(\operatorname{mon}(m, i-1)+\operatorname{mon}(m, i))$ nonlinear monomials. Hence,

$$
V_{L}(n)= \begin{cases}\sum_{i=0}^{2 m-2}(\operatorname{mon}(m, i)+\operatorname{mon}(m-1, i-1)) & \text { if } n=2 m \\ \sum_{i=0}^{2 m-1}(\operatorname{mon}(m, i-1)+\operatorname{mon}(m, i)) & \text { if } n=2 m+1\end{cases}
$$

Vector. In the following, $\mu(e)$ is the number of monomials in a parametric polynomial expression $e$ in normal form; $\kappa(e)$ is the number of constant monomials in $e(\kappa(e) \in\{0,1\}) ; \lambda(e)$ is the number of linear and non constant monomials in $e(\lambda(e) \in\{0,1\})$; and $\bar{\lambda}(e)$ is the number of nonlinear monomials in $e$. Clearly, $\mu(e)=\kappa(e)+\lambda(e)+\bar{\lambda}(e)$. Note that, since $\kappa, \lambda$, and $\bar{\lambda}$ are mutually exclusive, identifying $\mu(e)$ with one of them implies that the other are null. Finally, $\delta(e)$ is the common degree of all monomials in $e$ (or $\perp$ if it does not exist). A polynomial $\mathrm{P}_{n}(x)$ consists of parametric coefficients $\pi_{n, i}$ for $0 \leq i \leq n$, where $\pi_{n, n}=1$ (i.e., $\mu\left(\pi_{n, n}\right)=\kappa\left(\pi_{n, n}\right)=1$ and $\left.\delta\left(\pi_{n, n}\right)=0\right)$. If $n>0$ is even $(n=0$ is a particular case of the previous one), then for all $0 \leq i<n, \pi_{n, i}$ consists of a sum of $\mu\left(\pi_{n, i}\right)=\operatorname{mon}(n \div 2, i)$ monomials, all of them of degree $n-i$ (i.e., $\left.\delta\left(\pi_{n, i}\right)=n-i\right)$. Thus, $\pi_{n, i}$ is linear (and nonconstant) if $n-i=1$. Therefore, $\mu\left(\pi_{n, n-1}\right)=\lambda\left(\pi_{n, n-1}\right)$ and, for all $0 \leq i<n-1, \mu\left(\pi_{n, i}\right)=\bar{\lambda}\left(\pi_{n, i}\right)$ and $\delta\left(\pi_{n, i}\right)=n-i$. If $n$ is odd, then $\pi_{n, 0}=0$ and for all $0<i<n, \pi_{n, i}$ consists of a sum of $\mu\left(\pi_{n, i}\right)=\operatorname{mon}(n \div 2, i-1)$ monomials, all of them of degree $n-i+1$ (i.e., $\left.\delta\left(\pi_{n, i}\right)=n-i+1\right)$. Summarizing: $\mu\left(\pi_{n, i}\right)=\operatorname{mon}(n \div 2, i-(n \% 2))$. A constraint $P \geq 0$ is translated into a set of $n+1$ inequalities $C_{i} \geq 0$, where $C_{i}$ is the result of multiplying the $i$-th row of $M_{n}=\left(m_{i j}^{n}\right)_{n+1 \times n+1}$ and $[P]_{\mathcal{S}_{n}}$, the vector of coefficients of $P$, for $i=0, \ldots, n$. We have the following results. 
Proposition 5. For all $n, \mu\left(m_{1,2}^{n}\right)=0$ and for all $1 \leq j<i \leq n, \mu\left(m_{i, i}^{n}\right)=1$ and $\mu\left(m_{i, j}^{n}\right)=0$. Let $n>1$. For all $1 \leq i \leq n$,

$$
\begin{aligned}
& \text { 1. } \mu\left(m_{i, n+1}^{n}\right)=\sum_{j=1}^{n} \mu\left(m_{i j}^{n-1}\right) \mu\left(\pi_{n, j-1}\right)=\sum_{j=1}^{n} \mu\left(m_{i j}^{n-1}\right) \operatorname{mon}(n \div 2,(j-1)- \\
& n \% 2) \text {. } \\
& \text { 2. } \delta\left(m_{i, n+1}^{n}\right)=\delta\left(m_{i, n}^{n-1}\right)+1=n+1-i .
\end{aligned}
$$

Proposition 6. $V_{L}(0)=V_{L}(1)=0$ and for all $n>1, V_{L}(n)=V_{L}(n-1)+$ $\sum_{i=1}^{n-1} \mu\left(m_{i, n+1}^{n}\right)$.

\subsection{Comparison}

Let $V_{P}(n)=V(n)+V_{L}(n)$ be the number of parameters obtained after matching a given representation and issuing the preprocessing step LO for the linearization. The following table shows $V_{P}(n)$ for some degrees $n$ of the targeted polynomial $P$ for the considered representation methods ${ }^{3}$.

\begin{tabular}{|c|c|c|c|c|c|c|c|c|c|c|c|c|}
\hline Method & 1 & 2 & 3 & 4 & 5 & 6 & 7 & 8 & 9 & 10 & 20 & 100 \\
\hline Hilbert & 10 & 18 & 28 & 40 & 54 & 70 & 88 & 108 & 130 & 154 & 504 & 10504 \\
P\&S & 6 & 10 & 20 & 28 & 36 & 46 & 56 & 68 & 80 & 94 & 284 & 5404 \\
K\&S & 2 & 4 & 7 & 13 & 20 & 38 & 57 & 111 & 166 & 328 & 78741 & $9.57 \cdot 10^{23}$ \\
Vector & 0 & 2 & 6 & 28 & 96 & 498 & 2322 & 15308 & 93696 & 758086 & $2.48 \cdot 10^{16}$ & $<\infty$ \\
\hline
\end{tabular}

Although the range of values for $n$ is small, the trend for the different methods is clear and suggests that, for $n>6$, Pólya \& Szegö's representation provides the best starting point for an implementation. Let's reason that this is actually the case. Let $W_{L}(n)$ be the number of variables introduced by the linearization after using $L 0$ and $L 1, \ldots, L 3$. Obviously, $V_{L}(n) \leq W_{L}(n)$. Let $V_{T}(n)=V(n)+$ $W_{L}(n)$ be the number of variables occurring in the linear formula obtained by the linearization process. The number $F_{L}(n)$ of new formulas introduced by the linearization is bounded by $|D| W_{L}(n) \leq F_{L}(n)$. And the total number of formulas is $F_{T}(n)=I(n)+F_{L}(n)$, thus bounded by $I(n)+|D| W_{L}(n) \leq F_{T}(n)$.

Since the degree of all monomials in the parametric polynomials in the representation is 2, for Pólya and Szegö's representation $W_{L}^{P S}(n)=V_{L}^{P S}(n)$ (the linearization process will not introduce more variables after $L 0)$. Thus, $V_{T}^{P S}(n)=$ $V^{P S}(n)+V_{L}^{P S}(n)=V_{P}^{P S}(n)$. The $V_{L}^{P S}(n)$ equations $x_{M}=M$ are transformed by the application of $L 1$ or $L 2$ only (because $\operatorname{deg}(M)=2$ ) into $F_{L}^{P S}(n)=$ $|D| V_{L}^{P S}(n)$ new linear formulas. Thus, $F_{T}^{P S}(n)=I^{P S}(n)+|D| V_{L}^{P S}(n)$.

Since for $M \in\{$ Hilbert, KS, Vector, $G\}, V_{T}^{P S}(n)=V_{P}^{P S}(n)<V_{P}^{M} \leq V_{T}^{M}(n)$ for all $n>6$ (see the table above ${ }^{4}$ ), and, since $I^{P S}(n)<I^{M}(n)$ for all $n>1$, we have $F_{T}^{P S}(n)=I^{P S}(n)+|D| V_{L}^{P S}(n)<I^{M}(n)+|D| W_{L}^{M}(n) \leq F_{T}^{M}(n)$ for all $n>6$, we finally conclude that Pólya and Szegö's representation is the best choice for an implementation using the constraint solving method in [5]: it minimizes both the number of variables $V_{T}(n)$ and formulas $F_{T}(n)$ to be considered.

\footnotetext{
${ }^{3}$ Obtained using Haskell encodings of the cost formulas in Appendix B.

${ }^{4}$ Although we do not provide information about $V_{L}^{G}(n)$, note that $V^{G}(n)$ and $V_{T}^{P S}$ are already very similar. Thus, assuming $V_{T}^{P S}(n)<V_{T}^{G}(n)$ is natural.
} 


\section{$5 \quad$ Related work and conclusions}

In Section 3, we have shown that the notions of polynomial bases and vector coordinates can be used instead of that of monomials and monomial coefficients when testing univariate polynomials $P$ for $\operatorname{Psd}([0,+\infty))$ and $P d([0,+\infty))$. The quantitative analysis in the previous section, though, suggests that this new method is hardly useful in practice. We show its theoretical interest as improving on the use of Bernstein's polynomials [3], which inspired our developments.

$\operatorname{Psd}([0,+\infty))$ and $P s d([-1,1])$ are related through Goursat transform (see [14]): Given $P \in \mathbb{R}[X]$ of degree $n$, we let $\widetilde{P}(X)=(1+X)^{n} P\left(\frac{1-X}{1+X}\right)$. Furthermore, $\widetilde{\widetilde{P}}(X)=2^{n} P(X)$. Then, $P$ is $\operatorname{Psd}([-1,1])$ if and only if $\widetilde{P}$ is $\operatorname{Psd}([0,+\infty))$ and $\operatorname{deg}(\widetilde{P}) \leq n$, see $[14$, Lemma 1$]$. Testing $P d([-1,1])$ or $P s d([-1,1])$ of univariate polynomials $P \in \mathbb{R}[X]$ on $[-1,1]$ can be done by using the so-called Bernstein's basis $[6]$ : if $[P]_{\mathcal{B}_{n}}>\mathbf{0}$, for the Bernstein basis $\mathcal{B}_{n}$ (which consists of polynomials of degree $n$ only) then $P$ is $\operatorname{Pd}([-1,1])$ [2]. Unfortunately, $\mathcal{B}_{n}$ does not capture all $P \in P d([-1,1])$ as positive vectors $[P]_{\mathcal{B}_{n}}$. For instance, $P(X)=5 X^{2}-4 X+1$ is positive on $[-1,1]$ but $[P]_{\mathcal{B}_{2}} \ngtr \mathbf{0}[6]$. Nevertheless, for each $P \in \operatorname{Pd}([-1,1])$ of degree $n$ the so-called Bernstein's Theorem [4] ensures the existence of some $p \geq$ $n$ such that $[P]_{\mathcal{B}_{p}}$ consists of positive coordinates only (the minimum of those $p$ is called the Bernstein degree of $P$ ). Unfortunately, such $p$ can be much higher than $n$. For instance, for $P(X)=5 X^{2}-4 X+1$ ) we need to consider 23 polynomials in Bernstein's basis. Even worst, the Bernstein degree of a polynomial $P$ is not usually known, and we have to (over)estimate it. For instance, a the recent estimation [6] is $\frac{n(n-1)}{2} \frac{M}{\lambda}$, where $n$ is the degree of the polynomial, $M$ is the maximum value of the coordinates $[P]_{\mathcal{B}_{n}}$ of $P$ in the Bernstein basis of degree $n$, and $\lambda$ is the minimum of $P$ on $[-1,1]$. For $P(X)=5 X^{2}-4 X+1$ we have $n=2, M=10, \lambda=\frac{1}{5}$, and a estimation of 50, far beyond 23, the real Bernstein degree of $P$. In [6], this problem is addressed by using partitions of $[-1,1]$ where we are able to represent $P$ in a Bernstein basis of degree $n$ by using positive coordinates only. However, we need to produce several (up to $n+1$ ) partitions of $[-1,1]$, compute the corresponding representations of $P$, etc. Furthermore, it is unclear how [6] would be used with parametric polynomials (see Remark 1).

Example 7. For our running example, we get $\widetilde{Q}(X)=-10 X^{3}+4 X^{2}+10 X+4$. According to $[6$, page 640$]$, for $\mathcal{B}_{3}=\left\{\left(\begin{array}{l}3 \\ i\end{array}\right) \frac{(1-X)^{3-i}(X+1)^{i}}{8} \mid 0 \leq i \leq 3\right\}$, i.e., $\left\{\frac{1}{8}\left(1-3 x+3 x^{2}-x^{3}\right), \frac{3}{8}\left(1-x-x^{2}+x^{3}\right), \frac{3}{8}\left(1+x-x^{2}-x^{3}\right), \frac{1}{8}\left(1+3 x+3 x^{2}+x^{3}\right)\right\}$ we have: $S_{\mathcal{S}_{3} \mapsto \mathcal{B}_{3}}=\left(\begin{array}{cccc}1 & -1 & 1 & -1 \\ 1 & -\frac{1}{3} & -\frac{1}{3} & 1 \\ 1 & \frac{1}{3} & -\frac{1}{3} & -1 \\ 1 & 1 & 1 & 1\end{array}\right)$ and $[\widetilde{Q}]_{\mathcal{B}_{3}}=S_{\mathcal{S}_{3} \mapsto \mathcal{B}_{3}}[\widetilde{Q}]_{\mathcal{S}_{3}}=\left(\begin{array}{c}8 \\ -\frac{32}{3} \\ 16 \\ 8\end{array}\right)$, which does not witness $\widetilde{Q}$ as $P s d([-1,1])$ due to the negative coordinate $-\frac{32}{3}$ in 
$[\widetilde{Q}]_{\mathcal{B}_{3}}$. The estimated Bernstein degree (for $n=3, M=16$ and $\lambda \simeq 1.22$ ) is 40 , i.e, a 40-square cb-matrix is required! This can be compared with Example 5.

We have investigated methods for proving univariate polynomials $\operatorname{Ps}([0,+\infty))$, and a quantitative evaluation of the requirements needed to make a practical use of them suggests that an early result by Pólya and Szegö's provides an appropriate basis for implementations in most cases. An important motivation and contribution of this work in connection with the development of tools for automatically proving termination is that we avoid the need of explicitly requiring that parametric polynomials arising in proofs of termination have non-negative coefficients (which is the usual practice in termination provers, see $[8,12])$. We will use our new findings in future versions of the tool MU-TERM [1].

Acknowledgements. I thank the anonymous referees for their valuable comments.

\section{References}

1. B. Alarcón, R. Gutiérrez, S. Lucas, R. Navarro-Marset. Proving Termination Properties with MU-TERM. In Proc. of AMAST'10, LNCS 6486:201-208, 2011.

2. S. Basu, R. Pollack, and M.-F. Roy. Algorithms in Real Algebraic Geometry. Springer-Verlag, Berlin, 2006.

3. S. Bernstein. Démonstration du théorème de Weierstrass fondée sur le calcul des probabilités. Communic. Soc. Math. de Kharkow 13(2):1-2, 1912.

4. S. Bernstein. Sur la répresentation des polynômes positifs. Communic. Soc. Math. de Kharkow 14(2):227-228, 1915.

5. C. Borralleras, S. Lucas, A. Oliveras, E. Rodríguez, and A. Rubio. SAT Modulo Linear Arithmetic for Solving Polynomial Constraints. Journal of Automated Reasoning48:107-131, 2012.

6. F. Boudaoud, F. Caruso, and M.-F. Roy. Certificates of Positivity in the Bernstein Basis. Discrete Computational Geometry 39:639-655, 2008.

7. M.D. Choi, T.Y. Lam, and B. Reznick. Sums of squares of real polynomials. In Proc. of the Symposium on Pure Mathematics, vol. 4, American Mathematical Society, pages 103-126, 1995.

8. E. Contejean, C. Marché, A.-P. Tomás, and X. Urbain. Mechanically proving termination using polynomial interpretations. Journal of Automated Reasoning, 32(4):315-355, 2006.

9. D. Hilbert. Über die Darstellung definiter Formen als Summe von Formenquadraten. Mathematische Annalen 32:342-350, 1888.

10. H. Hong and D. Jakuš. Testing Positiveness of Polynomials. Journal of Automated Reasoning 21:23-38, 1998.

11. S. Karlin and W.J. Studden. Tchebycheff systems: with applications in analysis and statistics. Interscience, New York, 1966.

12. S. Lucas. Polynomials over the reals in proofs of termination: from theory to practice. RAIRO Theoretical Informatics and Applications, 39(3):547-586, 2005.

13. G. Polya and G. Szegö. Problems and Theorems in Analysis II Springer-Verlag, 1976.

14. V. Powers and B. Reznick. Polynomials that are positive on an interval. Transactions of the AMS 352(10):4677-4692, 2000.

15. V. Powers and T. Wörmann. An algorithm for sums of squares of real polynomials. Journal of Pure and Applied Algebra 127:99-104, 1998. 